\section{Case Reports in Neurology}

\title{
The Need for a Rational Approach to Vasoconstrictive Syndromes: Transcranial Doppler and Calcium Channel Blockade in Reversible Cerebral Vasoconstriction Syndrome
}

\author{
Elisabeth B. Marsh ${ }^{a}$ Wendy C. Ziai ${ }^{b}$ Rafael H. Llinas ${ }^{a}$ \\ ${ }^{a}$ Department of Neurology, The Johns Hopkins University School of Medicine,

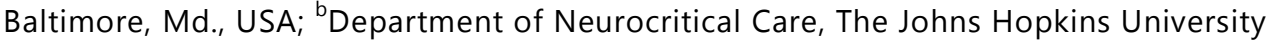 \\ School of Medicine, Baltimore, Md., USA
}

\section{Keywords}

Reversible cerebral vasoconstriction syndrome · Infarct · Thunderclap headache · Vasospasm . Transcranial Doppler ultrasound · Calcium channel blocker

\begin{abstract}
Introduction: Reversible cerebral vasoconstriction syndrome (RCVS) typically affects young patients and left untreated can result in hemorrhage or ischemic stroke. Though the disorder has been well characterized in the literature, the most appropriate way to diagnose, treat, and evaluate therapeutic response remains unclear. In previous studies, transcranial Doppler ultrasound (TCD) has shown elevated velocities indicative of vasospasm. This imaging modality is noninvasive and inexpensive; an attractive option for diagnosis and therapeutic monitoring if it is sensitive enough to detect changes in the acute setting given that RCVS often affects the distal vessels early in the course of disease. There is also limited data that calcium channel blockade may be effective in treating vasospasm secondary to RCVS, though the agent of choice, formulation, and dose are unclear. Methods: We report a small cohort of seven patients presenting with thunderclap headache whose vascular imaging was consistent with RCVS. All were treated with calcium channel blockade and monitored with TCD performed every 1-2 days. Results: On presentation, TCD correlated with standard neuroimag-
\end{abstract}


ing findings of vasospasm (on MR, $\mathrm{CT}$, and conventional angiography). TCD was also able to detect improvement in velocities in the acute setting that correlated well with initiation of calcium channel blockade. Long-acting verapamil appeared to have the greatest effect on velocities compared to nimodipine and shorter-acting calcium channel blockers. Conclusion: Though small, our cohort demonstrates potential utility of TCD to monitor RCVS, and relative superiority of extended-release verapamil over other calcium channel blockers, illustrating the need for larger randomized trials.

\section{Introduction}

Reversible cerebral vasoconstriction syndrome (RCVS) classically presents as a thunderclap headache that can progress to hemorrhage or infarction if left untreated. RCVS was first described in 1988 [1-4] and is characterized by reversible focal narrowing of the medium-sized intracranial vessels without evidence of inflammation within the cerebrospinal fluid. The mean age of onset is 42 and the disorder is more common in women [5]. It is seen most frequently in migraineurs and has been linked to triggers such as pregnancy, sexual intercourse, and vasoactive agents.

Though the clinical syndrome of RCVS has been relatively well characterized, the most appropriate way to diagnose, treat, and evaluate therapeutic response lacks support in the literature. In 2011, Singhal and colleagues [6] reported the largest series of patients with RCVS ( $n=139)$. In their cohort, the use of calcium channel blockade compared to no pharmacologic treatment did not significantly improve outcome 2-4 months after onset $(\mathrm{p}=$ 0.52). Unfortunately, the calcium channel blocker used, dose, and duration varied within the population so the significance of their results remains unclear. Both nimodipine and verapamil are common treatments [5]. Evidence for nimodipine is taken from the subarachnoid hemorrhage literature [7], while expert opinion has recommended oral verapamil [8]. There is no large, randomized controlled study that compares the efficacy of one agent, the formulation, or dose over another.

CT and MR, along with conventional angiography, have been used to diagnose RCVS and classically reveal a pattern of beading that resolves within weeks to months of the onset of headache. Transcranial Doppler ultrasound (TCD) has also shown elevated velocities on presentation that normalize over time [9]. In 2008, Chen and colleagues [10] used TCD measurement to follow vasoconstriction in 32 individuals presenting with RCVS. TCD was repeated every 10 days for the first month after the onset of headache and at 20-day intervals for 3 months. They showed prolonged elevation within the middle cerebral arteries (MCAs) compared to healthy controls at a mean 22 days from symptom onset. Typically, vascular assessment repeated months after symptom onset ultimately shows normalization of velocities; however, only single case reports have used TCD to monitor progression or resolution of the disease with response to treatment in the acute setting [11-14]. Numerous studies have shown the utility of TCD in monitoring vasospasm, but these were with respect to aneurysmal rupture [15-18]. As RCVS initially affects the distal vessels (manifesting early as convexal subarachnoid hemorrhage) and progressively works its way inward (ultimately resulting in large vessel infarction) [19], it is unclear whether TCD may be of the same utility for daily monitoring in these cases. Given that TCD is an inexpensive, noninvasive imaging modality, the ability to use this technology to diagnose and monitor response to treatment has major potential benefit. 


\section{Case Reports in Neurology}

We describe 7 patients presenting to our tertiary referral center with thunderclap headache diagnosed with RCVS. All were treated with calcium channel blockade and underwent serial TCD analysis to evaluate its utility in monitoring disease progression and its association with short-term clinical improvement. Following the cases, we discuss the utility of TCD in monitoring RCVS progression, and of long-acting verapamil over shorter-acting agents.

\section{Subjects and Methods}

Over a 15-month period, 7 patients presented with thunderclap headache and were diagnosed with RCVS. Patient characteristics are summarized in table 1. Patient data were collected and stored for use in publication as part of an IRB-approved clinical/research database. All patients consented to participation in this case series and a modified Rankin scale (mRS) (>90 days post event) was obtained at that time. Case 1 was lost to follow-up and therefore, specific details of her case were removed to prohibit identification.

All patients underwent neurovascular imaging (MRA, CTA, or conventional angiography) that confirmed the presence of beading in one or more vascular distribution. Four patients also underwent lumbar puncture to rule out potential mimics. Patients were followed over the course of their hospitalization with TCD. Serial neurological examinations, and pain assessments (0-10 Likert scale) completed by the nursing staff each shift were also performed.

All patients were treated with calcium channel blockade. The agent of choice and dosing were left up to the treating physician. In the majority of cases, patients also received intravenous magnesium and some form of pain management, though regimens differed.

Serial TCD was performed by the same examiner, using established methodology [20]. Evaluations were repeated every 1-2 days as determined by the primary team. Mean cerebral flow velocities through the bilateral middle, anterior, and posterior cerebral arteries (MCA, ACA, PCA) were recorded as well as the Lindegaard index. An MCA velocity of $>80$ $\mathrm{cm} / \mathrm{s}$ was considered abnormal and the diagnosis of vasospasm was determined using accepted criteria (mild: 100-140 cm/s, moderate: $140-200 \mathrm{~cm} / \mathrm{s}$, severe: $>200 \mathrm{~cm} / \mathrm{s}$ ) [17].

\section{Cases}

\section{Efficacy of Verapamil over Nimodipine}

Patient 1 is a young woman in her 20s who presented with a systolic blood pressure (SBP) of $>200 \mathrm{~mm} \mathrm{Hg}$, seizure, and right hemiparesis in the setting of thunderclap headache. She reported several weeks of worsening headaches in the setting of marijuana use. MRI showed multiple bilateral infarcts and MRA showed diffuse beading of the intracranial vessels that during angiography responded to intra-arterial injection of verapamil. A lumbar puncture was unremarkable. Following the angiogram, she was started on intravenous magnesium and nimodipine without change in her TCD velocities, which were elevated on presentation; however, velocities decreased when nimodipine was changed to verapamil, and normalized further when the extended-release form was used (see fig. 1). Verapamil SR was continued on discharge.

Outcome: discharged to acute rehabilitation with apraxia of speech and hemiparesis.

Follow-up mRS: unknown. 


\section{Case Reports in Neurology}

Patient 2 is a 54-year-old woman with a history of asthma, taking phenylephrine/dextromethorphan frequently for an upper respiratory infection. She developed 1-2 days of pain above her right eye, and presented to an outside hospital with thunderclap headache and left arm weakness. MRI showed a right parietal infarct and small subarachnoid hemorrhage. CTA showed poor filling in the bilateral ACA and MCA territories consistent with RCVS. A lumbar puncture was performed to rule out infection. She was started on nimodipine and transferred for further management. TCD revealed increased velocities bilaterally, most prominent in the left MCA. At day 4 after symptom onset, nimodipine was converted to long-acting verapamil dosed twice a day with subsequent improvement in TCD velocities. Verapamil SR was continued after discharge.

Outcome: discharged to acute rehabilitation with improving strength.

Follow-up 90 days: mRS: 0.

\section{Efficacy of Verapamil over Other Calcium Channel Blockers}

Patient 3 is a 36-year-old man with 'occasional headaches' who presented with a severe headache and right-sided weakness for 1 week, found to have a subacute stroke in the left basal ganglia. Blood pressure remained difficult to control throughout his hospitalization. Conventional angiogram showed only very mild irregularity of the left ACA. A stroke workup was unremarkable including lumbar puncture. His blood pressure was controlled and he was discharged home on an ACE inhibitor and beta-blocker. Five days later, he re-presented with recurrent thunderclap headache and SBP of $230 \mathrm{~mm} \mathrm{Hg}$ with a small subdural hematoma with subarachnoid along the right frontal convexity. He was started on nicardipine. RCVS was on the differential and TCD confirmed bilateral vasospasm. Two days later, longacting verapamil was initiated and over the next 2 days, he reported an improved pain score (7 on admission to 0 ), correlating with a reduction in velocities on TCD (see fig. 2). He was discharged on verapamil SR.

Outcome: discharged home with improved headaches.

Follow-up 90 days: mRS: 0.

Patient 4 is a 46-year-old previously healthy woman who presented to an outside hospital with thunderclap headache, nausea and subsequent generalized tonic clonic seizure. SBP was elevated (190 mm Hg). T2-weighted abnormalities suggestive of posterior reversible vasoconstrictive syndrome were seen on MRI. Lumbar puncture showed no evidence of inflammation and she was transferred for further workup. Conventional angiogram demonstrated mild proximal stenosis of multiple large vessels consistent with RCVS. TCD demonstrated mildly increased velocities, more pronounced on the left. Fibromuscular dysplasia was also seen and found to be present in the renal vessels, a potential trigger for her blood pressure elevation. She was initially started on nifedipine XL for blood pressure control without clinical improvement. After changing to oral verapamil (long acting administered once daily) her headaches began to improve though continued to occur immediately preceding her next scheduled dose. When twice a day dosing was initiated, her pain resolved. Verapamil SR was continued as an outpatient.

Outcome: discharged home with improved headaches.

Follow-up mRS: 0.

\section{Efficacy of Extended-Release over Short-Acting Verapamil}

Patient 5 is a 25 -year-old mother of 4 with a history of migraine who presented 12 days postpartum with a week of progressive headaches leading to the 'worst headache of her life' 


\section{Case Reports in Neurology}

and was found to have a right frontal intracerebral hemorrhage with evidence of beading on CTA and conventional angiogram consistent with postpartum angiopathy or RCVS. TCD confirmed elevated velocities in the bilateral MCAs. She was placed on short-acting verapamil with some improvement; however, when verapamil SR was given twice daily on day 5, normalization occurred (see fig. 1). Verapamil SR was continued on discharge.

Outcome: discharged home with near complete resolution of deficits.

Follow-up mRS: 1.

Patient 6 is a 37-year-old mother of 4 with a history of migraine who presented 5 days postpartum with a thunderclap headache and subsequent generalized tonic clonic seizure. Head CT showed a subarachnoid hemorrhage over the right frontal convexity and CTA showed subtle beading of the smaller branches of the bilateral ACA and MCA. MRI showed bilateral posterior T2 hyperintensities. She was started on short-acting verapamil and i.v. magnesium. As she improved and TCD velocities decreased, she was weaned off magnesium. She subsequently developed worsening of both clinical exam and velocities with diffuse vasospasm confirmed on MRA. Magnesium was restarted and she was converted to twice daily extended-release verapamil that was uptitrated. Over the next 4 days, velocities improved and eventually normalized prior to discharge. Verapamil SR was continued as an outpatient.

Outcome: discharged home neurologically intact with improved headache.

Follow-up mRS: 1.

\section{Treatment with Calcium Channel Blockade Hastens Recovery}

Patient 7 is a 37-year-old woman with a history of migraines, on celexa, who presented to an outside hospital with a thunderclap headache. A head CT showed no acute hemorrhage. She declined lumbar puncture. She received a triptan, after which time she became confused. Repeat imaging demonstrated a left frontal intracerebral hemorrhage with subarachnoid component and she was transferred for further management. A conventional angiogram was performed that was read as subtle beading in multiple vascular distributions. Elevated velocities were confirmed on TCD. She was started on twice daily extended-release verapamil and began to improve; however, there was disagreement as to the underlying diagnosis and verapamil was stopped. Velocities remained stable but did not improve and she was transferred out of the critical care unit. Four days later, her examination worsened. A CTA showed diffuse spasm throughout her intracranial vasculature, and TCD showed significantly increased velocities (see fig. 3). Repeat MRI showed a new stroke within the left MCA territory. Though the diagnosis of RCVS was now accepted, there remained disagreement over the best course of treatment. Due to the significant vasospasm and perfusion-dependent nature of her exam, the decision was made to augment blood pressure to maximize cerebral perfusion. Intravenous magnesium was continued. Slowly over the next couple of weeks, her velocities stabilized and she was able to be downgraded to the floor and ultimately discharged.

Outcome: discharged to an inpatient rehabilitation facility with significant aphasia, speaking nonsensically and only occasionally mimicking.

Follow-up mRS: 4.

\section{Discussion}

In each of the above cases, TCD was sensitive enough to detect elevated velocities and confirm the diagnosis of RCVS. In all instances, elevated velocities correlated with spasm 
seen on other neuroimaging modalities, and in case 2, TCD was actually more sensitive in detecting vasoconstriction. Our series also illustrates that in the acute period, serial TCD can document normalization of velocities and aid in following response to treatment. Contrary to the findings of Chen et al. [10], we saw velocities significantly decrease within days of initiation of calcium channel blockade. This discrepancy may be due to the fact that we found long-acting verapamil to be the most effective agent. The patients in the Chen series received nimodipine, which we found did not have the same velocity-lowering effect on TCD in our population. Case 7 supports this theory, as similar to the Chen series, when long-acting verapamil was not consistently administered, TCD velocities took weeks rather than days to normalize. This may also indicate that while the natural history of RCVS is resolution of spasm regardless of treatment, calcium channel blockade, and most specifically long-acting verapamil, hastens recovery.

TCD has the potential to be an attractive option for monitoring. It does not require radiation and is less expensive and invasive than the majority of other imaging techniques. Furthermore, it allows for efficient monitoring of the progression of disease and response to treatment. We demonstrate that the ability to follow velocities can aid in dose adjustment or aggressiveness of treatment and help prevent complications like those seen in case 7. Based on our experience, we recommend following TCD velocities until normalization and have demonstrated that with the use of verapamil, this may be shorter than initially described $[10,21]$. We choose to continue treatment through outpatient follow-up and determine longterm duration of calcium channel blockade on an individualized basis dependent on patient risk factors for recurrence and underlying comorbidities (e.g., hypertension, migraine).

In our population, extended-release verapamil prescribed twice daily appeared to most effectively decrease TCD velocities in the acute setting compared to other calcium channel blockers or no treatment. One could argue that verapamil was the second agent prescribed in the majority of our patients and that our observation was simply the natural resolution of the disease over time. However, there was a relatively convincing correlation between date of treatment initiation and decreasing velocities in many cases, and in others (e.g., case 7) when verapamil was stopped, velocities subsequently increased. Additionally, the use of twice daily long-acting verapamil also correlated with pain improvement in several cases. The effectiveness of extended-release verapamil prescribed twice daily makes sense if one assumes that mechanistically it leads to a more consistent steady state of drug level within the system. This observation supports the need for a multicenter, randomized controlled trial to determine the efficacy of verapamil over other agents.

There is a concern, as seen in case 7, that with severe diffuse vasospasm, the use of calcium channel blockade may result in an unsafe drop in blood pressure resulting in cerebral hypoperfusion and neurologic decline [21]. Our patients were monitored in a critical care setting with frequent neurological examinations. SBPs remained stable or decreased modestly, often in conjunction with improving TCD velocities, with the addition of extendedrelease verapamil and no patient experienced neurologic decline (table 1; fig. 1, 3). However, many patients were initially started on short-acting agents (nimodipine or verapamil), possibly as a conservative measure to ensure adequate perfusion prior to conversion to longeracting forms.

Our case series is not without limitations. It is composed of a small number of patients from a single institution. Furthermore, treatment was not randomized and results may have been confounded by other variables such as the use of intravenous magnesium or method of pain control. At our institution, i.v. magnesium is commonly used along with calcium channel blockade; eliminating it as a confounder for the agent chosen, but making it impossible to 
determine its individual effects. A larger, randomized controlled trial comparing extendedrelease verapamil to nimodipine, with and without i.v. magnesium, would be necessary to adequately address this question. Because the series was observational, the use of pain scales and TCD was also not standardized, though the majority of patients underwent serial TCD evaluation every 1-2 days. Finally, it remains possible that RCVS is actually a spectrum of disorders, some of which are 'benign' and may resolve on their own regardless of treatment. Our patients varied in the severity of illness and trigger of their disease, yet all improved in the acute setting with treatment. One explanation may be that the use of calcium channel blockers hastens recovery. Whether they also lead to improved long-term outcomes or simply shorter disease duration is unclear.

Despite the limitations, we feel that our cohort illustrates a few key concepts. First, TCD is an inexpensive, noninvasive technology that appears sensitive enough for use in both diagnosis and therapeutic monitoring in patients with RCVS; though we are not advocating at this point for its use over an initial MRA/CTA. Our series also suggests that extended-release verapamil given twice daily may be the most efficacious calcium channel blocker for reducing TCD evidence of vasospasm, does not lead to symptomatic hypotension when monitored in an appropriate setting, and may hasten resolution of RCVS. Our results agree with the literature that treatment of RCVS should be continued until a consistent flow decrement approaching normal is observed [10], but disagree that velocities typically remain elevated for weeks, and this may be due to our use of verapamil. Most notably, our data illustrate the need for a multicenter, randomized controlled trial to determine the optimal management of this potentially devastating but reversible syndrome.

\section{Statement of Ethics}

This study was approved by our institutional review board and patients provided informed consent for their participation in the case series.

\section{Disclosure Statement}

The authors have nothing to disclose.

\section{References}

1 Snyder BD, McClelland RR: Isolated benign cerebral vasculitis. Arch Neurol 1978;35:612-614.

-2 Call G, Fleming M, Sealfon S, et al: Reversible cerebral segmental vasoconstriction. Stroke 1988;19:1159-1170.

-3 Bogousslavsky J, Despland PA, Regli F, et al: Postpartum cerebral angiopathy: reversible vasoconstriction assessed by transcranial Doppler ultrasounds. Eur Neurol 1989;29:102-105.

4 Serdaru M, Chiras J, Cujas M, et al: Isolated benign cerebral vasculitis or migrainous vasospasm? J Neurol Neurosurg Psychiatry 1984;47:73-76.

Ducros A: Reversible cerebral vasoconstriction syndrome. Presse Med 2010;39:312-322.

6 Singhal AB, Hajj-Ali RA, Topcuoglu MA, et al: Reversible cerebral vasoconstriction syndromes. Arch Neurol 2011;68:1005-1012.

7 Barker FG, Ogilvy CS: Efficacy of prophylactic nimodipine for delayed ischemic deficit after subarachnoid hemorrhage: a metaanalysis. J Neurosurg 1996;84:405-414.

8 Sidorov EV, Feng W, Caplan LR: Stroke in pregnant and postpartum women. Expert Rev Cardiovasc Ther 2011;9:1235-1247. 
Marsh et al:: The Need for a Rational Approach to Vasoconstrictive Syndromes: Transcranial Doppler and Calcium Channel Blockade in RCVS

-9 Singhal AB, Caviness VS, Begleiter AF, et al: Cerebral vasoconstriction and stroke after use of serotonergic drugs. Neurology 2002;58:130-133.

10 Chen S-P, Fuh J-L, Chang F-C, et al: Transcranial color Doppler study for reversible cerebral vasoconstriction syndromes. Ann Neurol 2008;63:751-757.

11 Nowak DA, Rodiek So, Henneken S, et el: Reversible segmental cerebral vasoconstriction (Call-Fleming syndrome): are calcium channel inhibitors a potential treatment option? Cephalalgia 2003;23:218-222 Bogousslavsky J, Despland PA, Regli F, Dubuis PY: Postpartum cerebral angiopathy: reversible vasoconstriction assessed by transcranial Doppler ultrasounds. Eur Neurol 1989;29:102-105.

13 Kubo S, Nakata H, Tatsumi T, Yoshimine T: Headache associated with postpartum cerebral angiopathy: monitoring with transcranial color-coded sonography. Headache 2002;42:297-300.

$\$ 14$ Rivera L, Nekoovaght-Tak S, Saylor D, et al: Successful management of reversible cerebral vasoconstriction syndrome-induced intracerebral hemorrhage with minimally invasive surgery plus recombinant tissue-type plasminogen activator. J Neurol Stroke 2014;1:00030.

15 Proust F, Callonec F, Clavier E, et al: Usefulness of transcranial color-coded sonography in the diagnosis of cerebral vasospasm. Stroke 1999;30:1091-1098.

16 Lysakowski C, Walder B, Costanza MC, Tramer MR: Transcranial Doppler versus angiography in patients with vasospasm due to a ruptured cerebral aneurysm: a systematic review. Stroke 2001;32:2292-2298.

17 Aaslid R: Transcranial Doppler assessment of cerebral vasospasm. Eur J Ultrasound 2002;16:3-10.

18 Sloan MA, Alexandrov AV, Tegeler CH, et al: Assessment: transcranial Doppler ultrasonography: report of the Therapeutics and Technology Assessment Subcommittee of the American Academy of Neurology. Neurology 2004;62:1468-1481.

19 Ducros A, Boukobza M, Porcher R, Sarov M, Valade D, Bousser MG: The clinical and radiological spectrum of reversible cerebral vasoconstriction syndrome: a prospective series of 67 patients. Brain 2007;130:3091-3101.

20 Baumgartner RW: Transcranial color-coded duplex sonography. J Neurol 1999;246:637-647.

-21 Ducros A: Reversible cerebral vasoconstriction syndrome. Lancet Neurol 2012;11:906-917. 


\section{Effect of Calcium Channel Blockade on TCD and Blood Pressure}
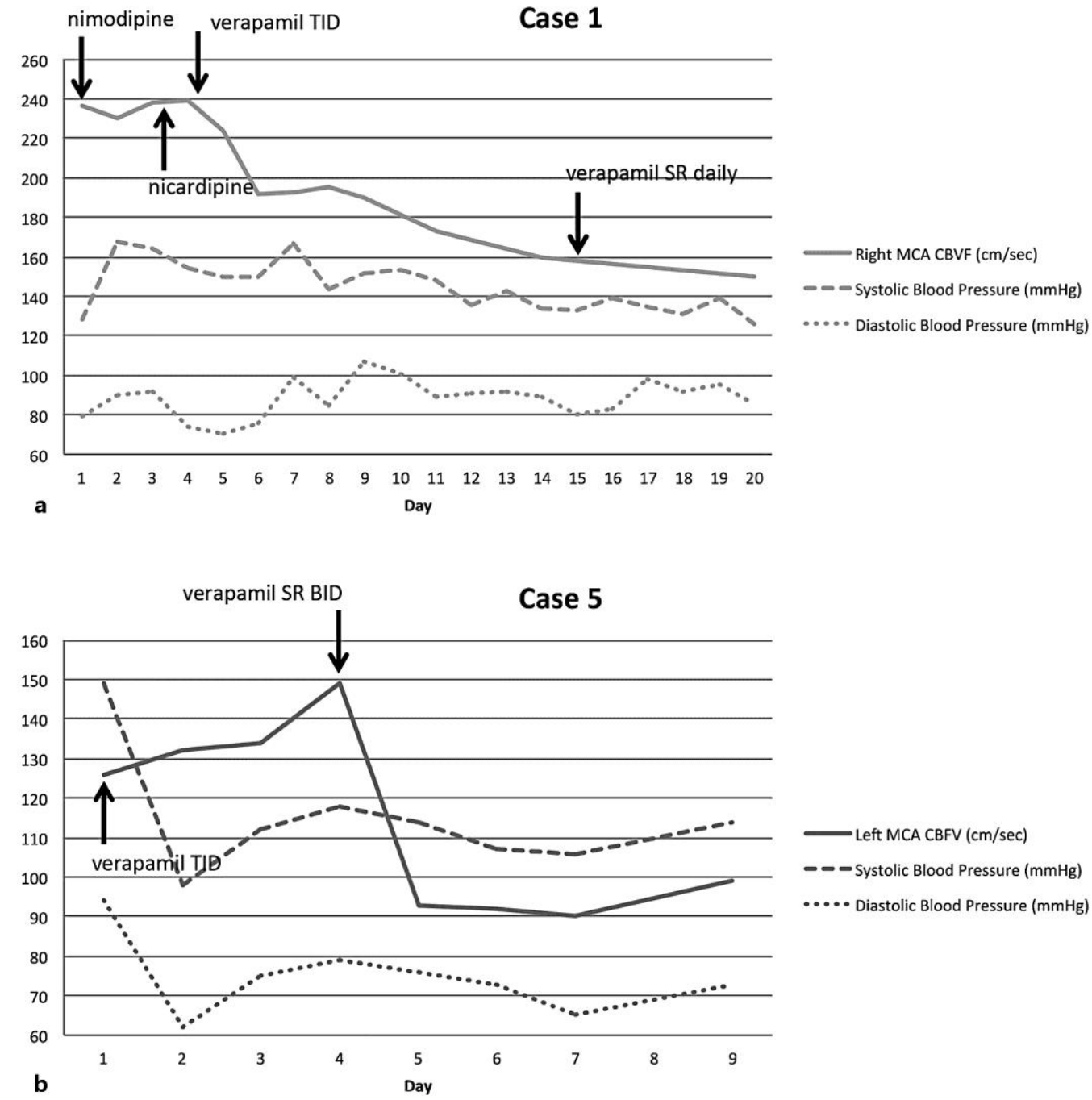

Fig. 1. Effect of calcium channel blockade on TCD and blood pressure. 

www.karger.com/crn

Marsh et al.: The Need for a Rational Approach to Vasoconstrictive Syndromes: Transcranial Doppler and Calcium Channel Blockade in RCVS



Fig. 2. Case 3: TCD velocities and pain.

\section{Case 7: Hospital Course by Treatment}

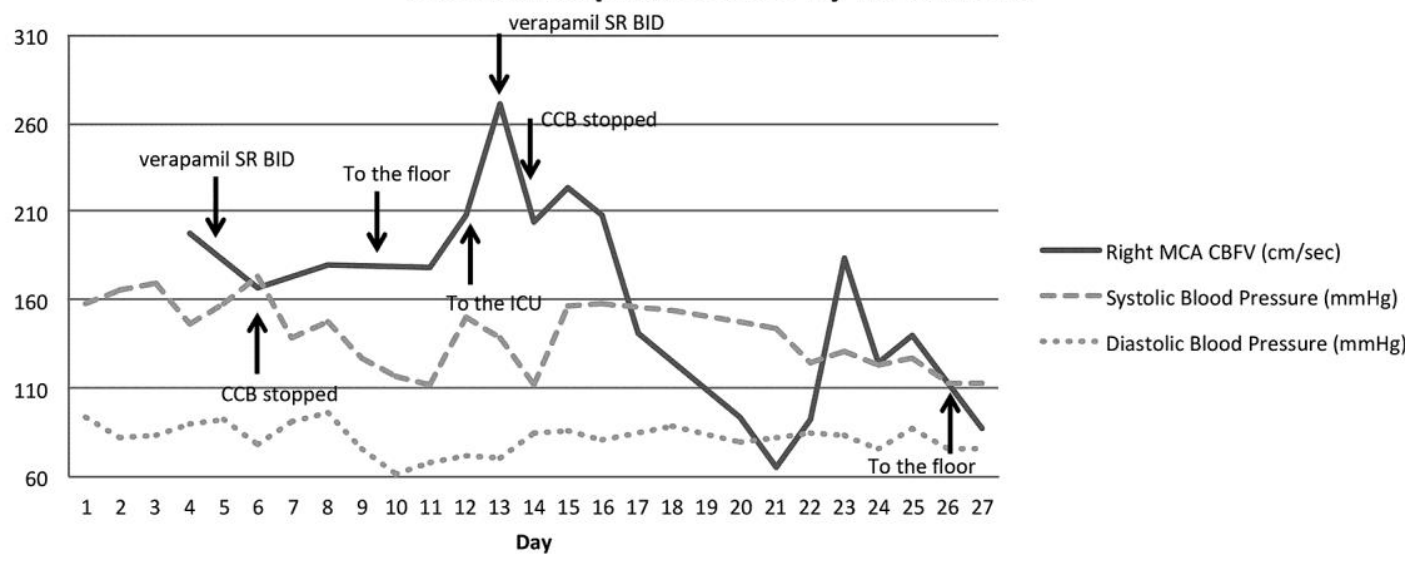

*CCB- calcium channel blocker

Fig. 3. Case 7: hospital course by treatment. 


\section{Case Reports in Neurology}

www.karger.com/crn

Marsh et al: The Need for a Rational Approach to Vasoconstrictive Syndromes: Transcranial Doppler and Calcium Channel Blockade in RCVS

Table 1. Patient characteristics

\begin{tabular}{|c|c|c|c|c|c|c|c|c|c|c|c|c|c|c|}
\hline \multirow[t]{2}{*}{ Case } & \multirow[t]{2}{*}{ Age } & \multirow[t]{2}{*}{ Race } & \multirow[t]{2}{*}{ Sex } & \multirow{2}{*}{$\begin{array}{l}\text { Mi- } \\
\text { graine } \\
\text { history }\end{array}$} & \multirow{2}{*}{$\begin{array}{l}\text { Precipi- } \\
\text { tating } \\
\text { factor }\end{array}$} & \multirow{2}{*}{$\begin{array}{l}\text { Clinical } \\
\text { presen- } \\
\text { tation }\end{array}$} & \multirow{2}{*}{$\begin{array}{l}\text { Initial } \\
\text { diag- } \\
\text { nostic } \\
\text { tool }\end{array}$} & \multicolumn{2}{|l|}{ Admission } & \multicolumn{2}{|c|}{ Treatment initiation } & \multicolumn{2}{|l|}{ Discharge } & \multirow{2}{*}{$\begin{array}{l}\text { Follow-up } \\
\text { mRS }\end{array}$} \\
\hline & & & & & & & & $\begin{array}{l}\text { blood } \\
\text { pressure }\end{array}$ & $\begin{array}{l}\text { mean } \\
\text { velocity }\end{array}$ & $\begin{array}{l}\text { blood } \\
\text { pressure }\end{array}$ & $\begin{array}{l}\text { mean } \\
\text { velocity }\end{array}$ & $\begin{array}{l}\text { blood } \\
\text { pressure }\end{array}$ & $\begin{array}{l}\text { mean } \\
\text { velocity }\end{array}$ & \\
\hline 1 & $20 \mathrm{~s}$ & $* *$ & $\mathrm{~F}$ & $* *$ & marijuana & $\begin{array}{l}\text { seizure, } \\
\text { bilateral } \\
\text { infarcts }\end{array}$ & MRA & $128 / 79$ & 236 & $128 / 79$ & 236 & $126 / 86$ & 150 & $* *$ \\
\hline 2 & 54 & $\mathrm{~W}$ & $\mathrm{~F}$ & no & $\begin{array}{l}\text { cough } \\
\text { suppressant }\end{array}$ & SAH & CTA & $135 / 85$ & $\begin{array}{l}\text { not per- } \\
\text { formed }\end{array}$ & $110 / 68$ & 142 & $116 / 72$ & 138 & 0 \\
\hline 3 & 36 & W & M & no & none & $\begin{array}{l}\text { basal } \\
\text { ganglia } \\
\text { ICH, SAH }\end{array}$ & TCD & $205 / 128$ & $\begin{array}{l}\text { not per- } \\
\text { formed }\end{array}$ & $177 / 99$ & 177 & $145 / 103$ & 103 & 0 \\
\hline 4 & 46 & W & $\mathrm{F}$ & no & hypertension & seizure & $\begin{array}{l}\text { angio- } \\
\text { gram }\end{array}$ & $165 / 69$ & $\begin{array}{l}\text { not per- } \\
\text { formed }\end{array}$ & $142 / 70$ & 108 & $128 / 76$ & $\begin{array}{l}\text { not per- } \\
\text { formed }\end{array}$ & 0 \\
\hline 5 & 25 & W & $\mathrm{F}$ & no & pregnancy & $\begin{array}{l}\text { right } \\
\text { frontal } \\
\text { ICH }\end{array}$ & СТA & $149 / 94$ & 126 & $149 / 94$ & 126 & $114 / 73$ & 99 & 1 \\
\hline 6 & 37 & other & $\mathrm{F}$ & yes & pregnancy & seizure & MRA & $131 / 70$ & $\begin{array}{l}\text { not per- } \\
\text { formed }\end{array}$ & $121 / 78$ & 140 & $107 / 68$ & 88 & 1 \\
\hline 7 & 37 & W & $\mathrm{F}$ & yes & triptan & $\begin{array}{l}\text { left } \\
\text { frontal } \\
\text { ICH }\end{array}$ & MRA & $158 / 93$ & $\begin{array}{l}\text { not per- } \\
\text { formed }\end{array}$ & $136 / 62$ & 198 & $109 / 67$ & 87 & 4 \\
\hline
\end{tabular}

$\mathrm{W}=$ White; $\mathrm{ICH}=$ intracerebral hemorrhage; $\mathrm{SAH}=$ subarachnoid hemorrhage. ${ }^{* *}$ Lost to follow-up; specific case details removed to prevent identification. 